\title{
Polar cap absorption events of November 2001 at Terra Nova Bay, Antarctica
}

\author{
L. Perrone, L. Alfonsi, V. Romano, and G. de Franceschi \\ Istituto Nazionale di Geofisica e Vulcanologia, Rome, Italy \\ Received: 12 September 2003 - Revised: 6 February 2004 - Accepted: 18 February 2004 - Published: 8 April 2004
}

\begin{abstract}
Polar cap absorption (PCA) events recorded during November 2001 are investigated by observations of ionospheric absorption of a $30 \mathrm{MHz}$ riometer installed at Terra Nova Bay (Antarctica), and of solar proton flux, monitored by the NOAA-GOES8 satellite in geo-synchronous orbit. During this period three solar proton events (SPE) on 4, 19 and 23 November occurred. Two of these are among the dozen most intense events since 1954 and during the current solar cycle (23rd), the event of 4 November shows the greatest proton flux at energies $>10 \mathrm{MeV}$. Many factors contribute to the peak intensity of the two SPE biggest events, one is the Coronal Mass Ejection (CME) speed, other factors are the ambient population of SPE and the shock front due to the CME. During these events absorption peaks of several $\mathrm{dB}(\sim 20 \mathrm{~dB})$ are observed at Terra Nova Bay, tens of minutes after the impact of fast halo CMEs on the geomagnetic field.

Results of a cross-correlation analysis show that the first hour of absorption is mainly produced by $84-500 \mathrm{MeV}$ protons in the case of the 4 November event and by $15-44 \mathrm{MeV}$ protons for the event of 23 November, whereas in the entire event the contribution to the absorption is due chiefly to $4.2-$ $82 \mathrm{MeV}$ (4 November) and by 4.2-14.5 MeV (23 November). Good agreement is generally obtained between observed and calculated absorption by the empirical flux-absorption relationship for threshold energy $E_{0}=10 \mathrm{MeV}$. From the residuals one can argue that other factors (e.g. X-ray increases and geomagnetic disturbances) can contribute to the ionospheric absorption.
\end{abstract}

Key words. Ionosphere (Polar Ionosphere, Particle precipitation) - Solar physics (Flares and mass ejections)

Correspondence to: L. Perrone

(perrone@ingv.it)

\section{Introduction}

In the 1940's and 1950's the emissions of solar protons associated with solar flares, as particles in many cases, leave the Sun within a few minutes after the solar flare's maximum (Cane et al., 2002). Recently, the role of Coronal Mass Ejections (CMEs) in producing major solar proton emission was emphasised to the point that a small group of solar particle physicists consider major solar proton events (SPE) to be completely independent of flares (Kahler, 2001; Reames, 1999). But Cane (2003) observed that particles with energies above $25 \mathrm{MeV}$ are consistent with a population of flare particles in major SPEs, whereas particles events associated with fast shocks, driven by fast CMEs, have the highest intensity below $20 \mathrm{MeV}$. The occurrence of SPE during minimum solar activity is very low, while in active Sun years, especially during the falling and rising phase of the solar cycle, the SPEs may average one per month.

It is well recognised that these solar particles have prompt and nearly complete access to the polar atmosphere via magnetic field lines interconnected between the interplanetary medium and the terrestrial field (van Allen et al., 1971). Consequently, they cause excess ionisation in the ionosphere, particularly concentrated in the polar cap, which, in turn, leads to an increase in the absorption of HF radio waves, termed polar cap absorption (PCA).

The ionisation occurs at various depths which depends on the incident particle energies, so that the ionisation in the D-region during PCA events is due mainly to protons with energy in the range of 1 to $100 \mathrm{MeV}$ that corresponds to an altitude between 30-80 km (Ranta et al., 1993; Sellers et al., 1977; Collis and Rietveld, 1990; Reid, 1974). Particles with even greater energies $(>500 \mathrm{MeV})$ are recorded on the ground by a cosmic-ray detector; these events are called Ground Level Enhancement (GLE) (Davies, 1990).

The major PCA events are associated with solar flares located on the side of the solar central meridian towards which the Sun rotates, that is, on the west side. It has also been found that the delay between flare outbreak and the start of 
a PCA depends mainly upon the heliographic latitude (Ranta et al., 1993). The boundary of the PCA region is typically between $60^{\circ}$ and $65^{\circ}$ geomagnetic latitude, while the durations of PCAs vary from a few hours to many days (Collis and Rietveld, 1990).

A characteristic feature of PCA events is the large difference between day and night absorption intensities for constant precipitating fluxes of solar particles. A day-to-night ratio in absorption intensities of around 4-8 is often observed during PCA events (Stauning, 1996; Hargreaves et al., 1993; Ranta et al., 1995; Pietrella et al., 2002). The most plausible explanation is a drastic increase in the effective recombination rate after sundown, i.e. when negative ions can exist and positive ions are mostly in the form of clusters which have much larger recombination rates than molecular ions usually found at higher altitudes, and during the day lowering the density of free electrons which cause ionospheric absorption. Speculations that the particles causing PCA were protons of solar origin were suggested before they could be verified by in situ experiments (Reid and Collins, 1959). Modern instruments carried on geostationary satellites are now able to provide continuous measurements of solar particles fluxes and their energy spectra. Routine monitoring of ionospheric absorption is possible since the riometric technique was introduced (Little and Leinbach, 1959). This instrument measures the amount of cosmic noise absorbed by the ionosphere at operating frequencies in the range $20-50 \mathrm{MHz}$.

During daytime when PCA event ionisation conditions exist, the dependence of electron density $N_{e}$ on ionisation rate $q$ is relatively simple; an excellent correlation has been observed between simultaneous measurements of HF absorption and proton fluxes. This prompted the development of empirical formulas connecting these two quantities for estimating one from the other when only a single measurement is available. When a daytime PCA occurs the absorption A (measured in $\mathrm{dB}$ ) should be proportional to the square root of the proton intensity, $\mathrm{A} \sim J^{1 / 2}$ (van Allen et al., 1964, Patterson et al., 2001).

In the current solar cycle 23 there occurred five severe SPEs $(>10000 \mathrm{pfu}$; pfu=particle flux units), five strong ( $>1000 \mathrm{pfu}), 19$ moderate ( $>100 \mathrm{pfu})$ and 43 (>10 pfu) minor intensity. Ranta et al. (1993) found that only the medium to strong events ( $>100 \mathrm{pfu}$ ) lead to appreciable variations in the absorption. In fact, SPE with pfu $<100$ result in variations of daily absorption below $2 \mathrm{~dB}$.

Three PCAs recorded at Terra Nova Bay, and SPE events during November 2001 (daylight period) are investigated. Two of these SPEs are severe events and one is of minor intensity. Owing to the continuous daylight over Antarctica the absorption data considered here do not show any day/night changes.

A cross-correlation analysis is applied to the ionospheric absorption (1-min data) by $30 \mathrm{MHz}$ riometer measurements and to the integral/differential solar protons flux (5-min data) by the NOAA-GOES-8 satellite. In Sects. 2 and 3 the data analysis are described and the preliminary results are shown and discussed. Finally, discussion and conclusions are outlined in Sect. 4.

\section{Observations}

In November 2001 the riometers installed at the Italian station of Terra Nova Bay (TNB) in Antarctica (geographic coordinates: $74.69^{\circ} \mathrm{S}, 164.12^{\circ} \mathrm{E}$; geomagnetic co-ordinates: $77.32^{\circ} \mathrm{S}, 278.92^{\circ} \mathrm{E}$ ) recorded three PCA events.

A solid state riometer (La Jolla; Chivers, 1975), at $30 \mathrm{MHz}$, had been placed at TNB in November 1994, to measure the cosmic noise coming from the outer space. The $30 \mathrm{MHz}$ measure is sampled every $1 \mathrm{~min}$. For calculating the ionospheric absorption it is necessary to determine the socalled Quiet Day Curve (QDC). The estimation of the QDC has been carried out first by taking into account the $85 \%$ of the maximum value of cosmic radio noise at every given sidereal time, and then by applying a Fourier expansion to these percentage values, in order to eliminate the data scattering at the same sidereal time (De Franceschi et al., 1997). Beside the riometer data for investigating the PCA events we have also analysed magnetospheric and solar parameters.

For this scope the following data are taken into account:

- The North Polar Cap index (PC) 1-min data recorded at Thule (geographic co-ordinates: $77.50^{\circ} \mathrm{N}, 290.80^{\circ} \mathrm{E}$, geomagnetic co-ordinates: $88.8^{\circ} \mathrm{N}, 12.5^{\circ} \mathrm{E}$ ). Using the Polar Cap index from the Northern Hemisphere is inevitable, since the index from Antarctic station Vostok, at the time of this analysis, is not available for the period considered. Generally, PC values greater than 12 indicate extremely disturbed periods (Lukianova et al., 2002).

- The 5-min integral solar proton flux, defined as the solar proton flux greater than a threshold energy $E_{0}$, and differential solar proton flux, defined as a solar proton flux with energy included between a lower and an upper limit. These data are derived by the NOAA GOES-8 satellite in geo-synchronous orbit recorded at seven different energy channels.

- Solar X-rays from 0.1-0.8 nm, measured by GOES-10 station with a 1-min sampling by the NOAA-GOES-10 satellite in geosynchronous orbit.

A full as possible description of PCA events is given which also considers the solar sources, as CMEs and flares, and the solar protons events linked to the PCAs. A list of these events and their characteristics is reported in Table 1. The starting time of PCA is considered when the ionospheric absorption is greater than $0.5 \mathrm{~dB}$, also the data about CME are obtained by SOHO's (Solar and Heliospheric Observatory) LASCO (Large Angle and Spectrometric Coronograph experiment) coronograph.

The temporal behaviour of both ionospheric absorption and differential proton flux is shown in Fig. 1. X-ray flux 
Table 1. List of the principal geophysical conditions during November 2001.

\begin{tabular}{|c|c|c|c|}
\hline Event & Starting time & Maximum time & Ending time \\
\hline \multicolumn{4}{|c|}{ First event } \\
\hline Solar Flare X1/3b burst II/IV(N06W18) & 4 November 16:03 UT & 4 November $16: 20 \mathrm{UT}$ & 4 November 16:47 UT \\
\hline Halo CME with a speed of $1810 \mathrm{~km} / \mathrm{s}$ & 4 November 16:35 UT & / & / \\
\hline Interplanetary shock due to the CME & 6 November 01:50 UT & / & / \\
\hline $\operatorname{SPE}(E>10 \mathrm{MeV})$ & 4 November 17:05 UT & $\begin{array}{l}6 \text { November } 02: 15 \mathrm{UT} \\
(\mathrm{max}=31700 \mathrm{pfu})\end{array}$ & 10 November 07:15 UT \\
\hline PCA & 4 November 16:55 UT & 6 November 02:00 UT & 9 November $02: 43 \mathrm{UT}$ \\
\hline \multicolumn{4}{|l|}{ GLE occurred on 4 November } \\
\hline \multicolumn{4}{|c|}{ Second event } \\
\hline Solar Flare M2.8/1n burst II/IV(S13E42) & 17 November 04:49 UT & 17 November 05:25 UT & 17 November 06:11 UT \\
\hline Halo CME with a speed of $1379 \mathrm{~km} / \mathrm{s}$ & 17 November 05:30 UT & / & / \\
\hline Interplanetary shock due to the CME & 19 November 17:00 UT & / & / \\
\hline $\operatorname{SPE}(E>10 \mathrm{MeV})$ & 19 November 12:30 UT & $\begin{array}{l}20 \text { November } 00: 10 \mathrm{UT} \\
(\max =34 \mathrm{pfu})\end{array}$ & 10 November 14:10 UT \\
\hline PCA & 19 November 21:37 UT & 20 November $01: 17$ UT & 20 November 04:31 UT \\
\hline \multicolumn{4}{|c|}{ Third event } \\
\hline Solar Flare M3.8/2b burst II/IV(S25W67) & 22 November 20:18 UT & 22 November 20:35 UT & 22 November 20:52 UT \\
\hline Halo CME with speed $1443 \mathrm{~km} / \mathrm{s}$ & 22 November 20:30 UT & / & / \\
\hline Solar Flare M9.9/2n burst II/IV(S15W34) & 22 November $22: 32$ UT & 22 November 23:30 UT & 23 November 00:06 UT \\
\hline Halo CME with speed $1437 \mathrm{~km} / \mathrm{s}$ & 22 November 23:30 UT & / & / \\
\hline Interplanetary shock due to the two CME's & 24 November 05:40 UT & l & I \\
\hline $\operatorname{SPE}(E>10 \mathrm{MeV})$ & 22 November 23:20 UT & $\begin{array}{l}24 \text { November 05:55 UT } \\
(\mathrm{max}=18900 \mathrm{pfu})\end{array}$ & 28 November 01:00 UT \\
\hline PCA & 23 November 00:25 UT & 24 November 06:00 UT & 26 November 13:09 UT \\
\hline
\end{tabular}

data and the geomagnetic polar cap index, PC, are also plotted for a better description of the solar-magnetic activity during November 2001.

The first PCA analysed here starts on 4 November and ends on 9 November (Table 1, Fig. 1). The proton flux enhancement begins at the highest energy band when the flare is still in progress. While the starting time of PCA is observed a few minutes later a proton flux enhancement in the band $84-500 \mathrm{MeV}$ occurs, simultaneous with the band 15$82 \mathrm{MeV}, 0.6-4.2 \mathrm{MeV}$ and about 20-35 min after the solar sources (Fig. 2). The arrows in Fig. 2 indicate the ambient intensity observed for the different solar proton energies just preceding the onset of the SPE. The PC index shows a moderate magnetic activity between 08:00-14:00 UT and between 16:00-18:00 UT. The ionospheric absorption beginning on 4 November decreased when four M Solar Flares $\left(10^{-5} \leq \mathrm{I}<10^{-4} \mathrm{~W} / \mathrm{m}^{2}\right)$ occurred on 5 November. This is probably responsible for a further increase in the proton flux after 16:00 UT and a further increase is observed after 20:00 UT (Fig. 3), whereas the PC has a fluctuating behaviour between 14:00-21:00 UT and definitively increases after 21:00 UT.

On 6 November an interplanetary shock due to the CME generated on 4 November is observed at 01:50 UT and as a probable consequence of this shock the maximum of the event is recorded around 02:00 UT and the PC reaches a value of 30 (Fig. 4). All energy bands show a proton flux increase around 02:00 and 03:00 UT, while another increase is observed between 04:00-06:00 UT, especially for the lower energy protons, and a decrease around 06:00 and 07:00 UT. After this peak the absorption starts to decline. The SPE and PCA decrease slowly, probably by a series of $\mathrm{M}$ solar flares occurring between 6-8 November (Fig. 1), which have enhanced the solar proton flux and X-ray flux.

A moderate PCA began on 19 November and ended on 20 November (Fig. 5). This event is characterised by a flare coming from the Sun's eastern hemisphere (Table 1). Compared to the first event the ambient intensity of SPE is greater for the energy band 0.6-44 MeV (Fig. 5). At 17:00 UT on 19 November an interplanetary shock is observed, associated with the CME (halo 05:30) which occurred on 17 November and the protons with energies between 0.6 and $14.5 \mathrm{MeV}$ show an increase between 18:00 UT and 20:00 UT. The highest energy band remains undisturbed. The ionospheric absorption further increases around 18:00 UT and the magnetic activity is disturbed, as indicated by PC values among 2.5 and 3.5 after 13:00 UT and up to 4 at 20:00 UT. Around 01:00 UT on the 20 November proton event, PCA and PC index reach their maximum (Fig. 5). 

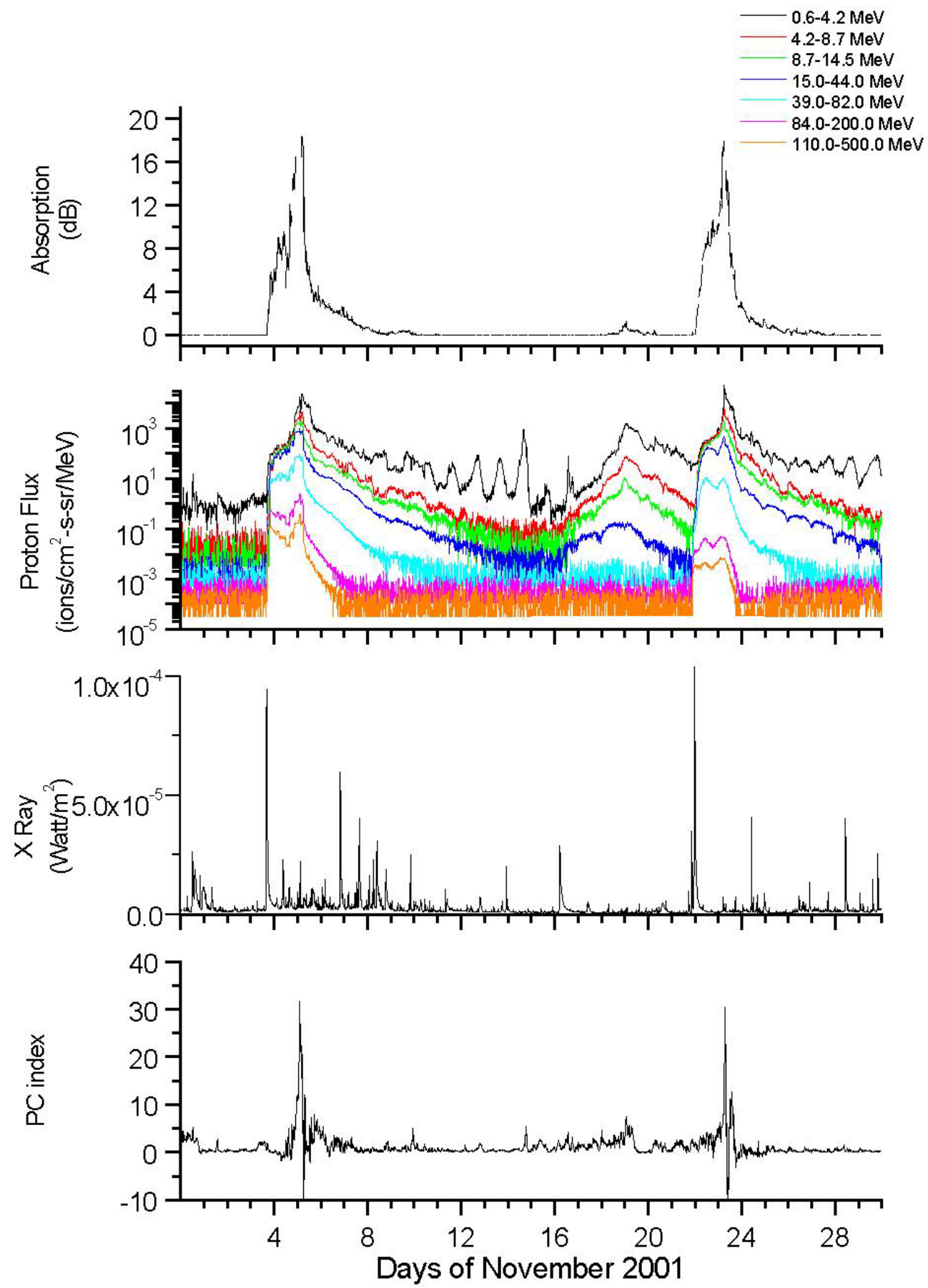

Fig. 1. Variation of ionospheric absorption, differential solar protons flux, solar X-rays (0.1-0.8 nm), PC index during November 2001 (from top to bottom).

The third Solar Proton Event analysed here starts on 22 November and ends on 26 November (Table 1). Compared to the first event the ambient intensity of SPE is greater for the energy band $0.6-14.5 \mathrm{MeV}$, in comparison to the second, for the energy bands of $0.6-4.2 \mathrm{MeV}$ and $8.7-14.5 \mathrm{MeV}$ (Fig. 6). On 22 November protons with $84-500 \mathrm{MeV}$ begin to grow after 21:00 UT, about $10 \mathrm{~min}$ after the first solar flare, whereas protons with energies between $0.6-8.7 \mathrm{MeV}$ are undisturbed. The PC index shows a moderate magnetic activity. The PCA starts at 0:25 UT on 23 November, around $3 \mathrm{~h}$ later than a proton flux of higher energy and from 1 to $4 \mathrm{~h}$ after the solar sources (Fig. 7). The PC is disturbed the whole day and reaches a value of 5 at 23:50 UT.

The PCA and SPE maxima on 24 November at about 06:00 UT are probably connected to a shock impacting the geomagnetic field at 05:40 UT (Fig. 8). This shock comes from the interaction between two interplanetary shock waves by the two CMEs on 22 November (Table 1). 


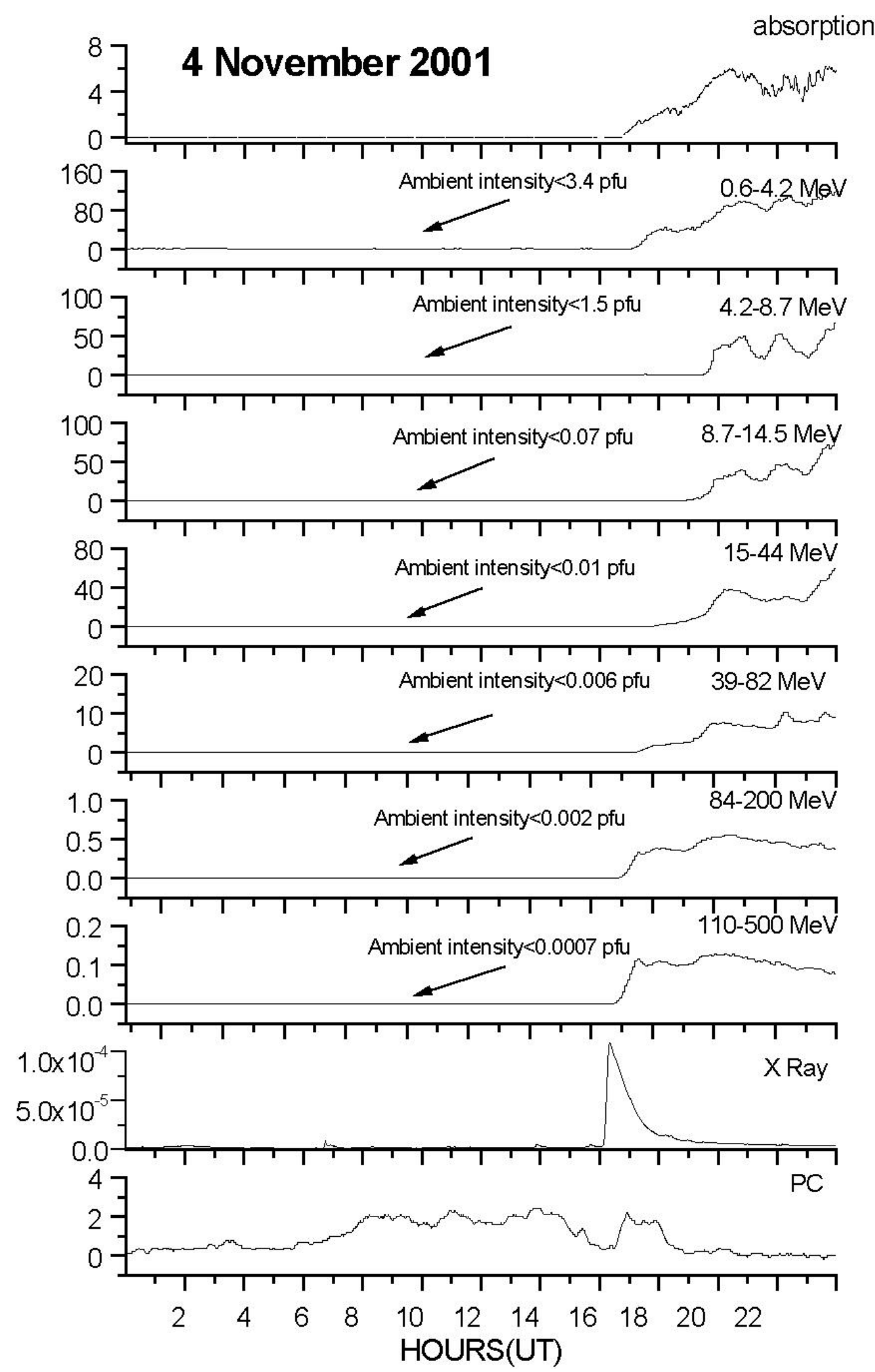

Fig. 2. As Fig. 1, but for 4 November. 


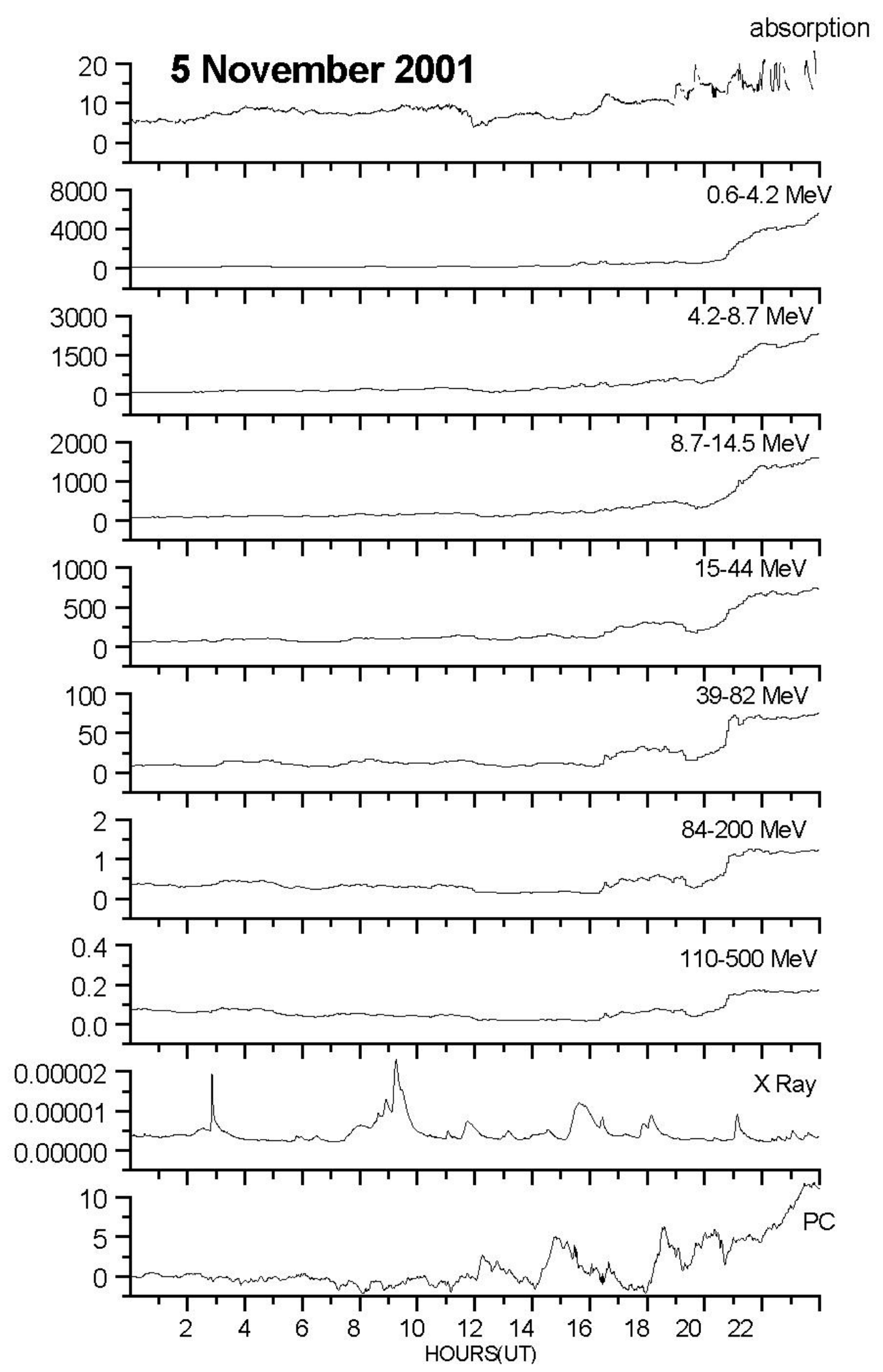

Fig. 3. As Fig. 1, but for 5 November. 
Table 2. Daily correlation coefficients between $A$ and $J^{1 / 2}$ for each differential protons flux energy band.

\begin{tabular}{|c|c|c|c|c|c|c|c|}
\hline Day of November & $\begin{array}{r}r \\
r\end{array}$ & $\begin{array}{c}\mathrm{r} \\
\mathrm{g}\end{array}$ & $\begin{array}{c}r \\
07\end{array}$ & $\stackrel{\mathrm{r}}{\mathrm{seV}}$ & $\begin{array}{r}r \\
30-82\end{array}$ & $\begin{array}{r}r \\
84-200 \mathrm{MeV}\end{array}$ & $\begin{array}{r}r \\
110-500 \mathrm{MeV}\end{array}$ \\
\hline 4 & 0.96 & 0.94 & 0.92 & 0.97 & 0.95 & 0.90 & 0.45 \\
\hline 5 & 0.58 & 0.84 & 0.73 & 0.57 & 0.66 & 0.26 & -0.015 \\
\hline 6 & 0.95 & 0.98 & 0.98 & 0.96 & 0.93 & 0.92 & 0.93 \\
\hline 7 & 0.81 & 0.84 & 0.87 & 0.86 & 0.95 & 0.96 & 0.91 \\
\hline 8 & 0.78 & 0.76 & 0.92 & 0.95 & 0.97 & 0.79 & 0.26 \\
\hline 9 & -0.11 & 0.82 & 0.90 & 0.91 & 0.90 & -0.28 & -0.30 \\
\hline 19 & 0.96 & 0.97 & 0.95 & 0.56 & 0.01 & -0.35 & 0.03 \\
\hline 20 & 0.92 & 0.91 & 0.90 & 0.86 & 0.21 & 0.11 & 0.01 \\
\hline 23 & 0.91 & 0.97 & 0.97 & 0.95 & 0.83 & 0.70 & 0.44 \\
\hline 24 & 0.78 & 0.97 & 0.97 & 0.96 & 0.86 & 0.82 & 0.81 \\
\hline 25 & 0.69 & 0.87 & 0.98 & 0.89 & 0.99 & 0.63 & -0.31 \\
\hline 26 & 0.68 & 0.95 & 0.91 & 0.74 & 0.90 & -0.19 & -0.37 \\
\hline
\end{tabular}

This interaction, plus the higher SPE ambient population at the time of the fast halo CME (Kalher, 2001), could be responsible for the enhancement of the peak intensity observed in the energy range $0.6-14.5 \mathrm{MeV}$ (Fig. 6). Compared to the first event the SPEs maxima are higher for the energy bands 0.6-8.7 MeV, for $8.7-14.5 \mathrm{MeV}$ they are similar, while they are smaller for the highest energy band. The PC index indicates storm conditions between 06:00 and 08:00 UT with a maximum of 29.

\section{Data analysis}

\subsection{Proton flux at commencement of PCA events}

For estimating the proton energy band mainly contributing to the initial phase of PCA, a cross-correlation analysis between the ionospheric absorption $A(t)$ and differential solar proton flux $J^{1 / 2}(t-\tau)$ for a different time delay ( $\tau=0,-5, \ldots-60 \mathrm{~min}$ ) is carried out for the two strongest events. In this analysis we use 5-min data of $A$ and $J$ between 16:55 UT and 17:55 UT (first hour of the absorption) of 4 November. $A(t)$ is well correlated with $84-500 \mathrm{MeV}$ solar protons with a delay between 0 and $30 \mathrm{~min}$. The highest correlations $r$ are found for the energy band 84-200 MeV $(r=0.99)$ and for $110-500 \mathrm{MeV}(r=0.98)$ for a time delay equal to 5 to $15 \mathrm{~min}$, while the correlation peak related to the energy band $0.6-4.2 \mathrm{MeV}$ is recorded for $\tau=0 \quad(r=0.98)$ (Fig. 9). Hence, the starting phase of the absorption is due mainly to $84-500 \mathrm{MeV}$ and $0.6-4.2 \mathrm{MeV}$.

For the event of 23 November $A$ and $J$ data between 0:25 UT and 1:25 UT (first hour of PCA) are considered. On 23 November (Fig. 10), protons of 15-44 MeV mainly contribute to the absorption for $\tau=-210, \ldots, 0$. For the energy band $110-500 \mathrm{MeV}$ the correlation coefficient is $\geq 0.9$ for $\tau=-205, \ldots,-195 \mathrm{~min}$ (Fig. 10). In this case the starting phase of the absorption is due mainly to protons with energies between $15-44 \mathrm{MeV}$.

\subsection{Proton flux throughout the PCA event}

The daily impact of the differential solar proton flux on the lower ionosphere is also investigated. A correlation analysis is then applied between $A$ and $J^{1 / 2}$ hourly means along the days marked by the three SPE/PCA events. The results are presented in Table 2.

On 4 November a very high correlation $(r \geq 0.9)$ is found for the protons with energies between $0.6-200 \mathrm{MeV}$, with a peak in the range 15 to $44 \mathrm{MeV}$, whereas $110-500 \mathrm{MeV}$ protons don't seem to be correlated with the absorption. On 5 November the correlation is quite high $(r=0.73-0.84)$ only for the energy band 4.2-14.5 MeV. For the day of the maximum (6 November), the correlation is very high for the entire energy band ( $r=0.92-0.98)$ and the peak is in the range 4.2$14.5 \mathrm{MeV}(r=0.98)$. After the maximum of the absorption, the correlation slowly decreases, depending on the different protons energies. During the entire event (4-9), the main contribution is from protons with energies 4.2 to $82 \mathrm{MeV}$ penetrating to altitudes between 80 and $40 \mathrm{~km}$.

From 19 to 20 November protons with lower energies $(0.6-14.5 \mathrm{MeV})$ are mainly involved, indicating that the absorption is produced principally at a higher altitude region than in the previous case $(>70 \mathrm{~km})$. The daily correlation for 23 November results $>0.9$ for $0.6-44 \mathrm{MeV}$ protons, while on the peak day ( 24 November) the results show $r>0.9$ for the energy band 4.2-44 MeV (Table 2). During the entire event (23-26), the main contribution was from protons with energies of 4.2-14.5 MeV. It means that the primary contribution to the PCA arises from ionospheric regions between 80 and $60 \mathrm{~km}$.

From the analysis of cross-correlation and the correlation between $A$ and $J^{1 / 2}$ it can thus be argued that for the first event the starting phase of the absorption is due to protons of $84-500 \mathrm{MeV}$ and $0.6-4.2 \mathrm{MeV}$ (Fig. 9), whereas only in the later phase, between 19:00 UT and 20:00 UT the other channels go into action (see Fig. 2). For the third event the 


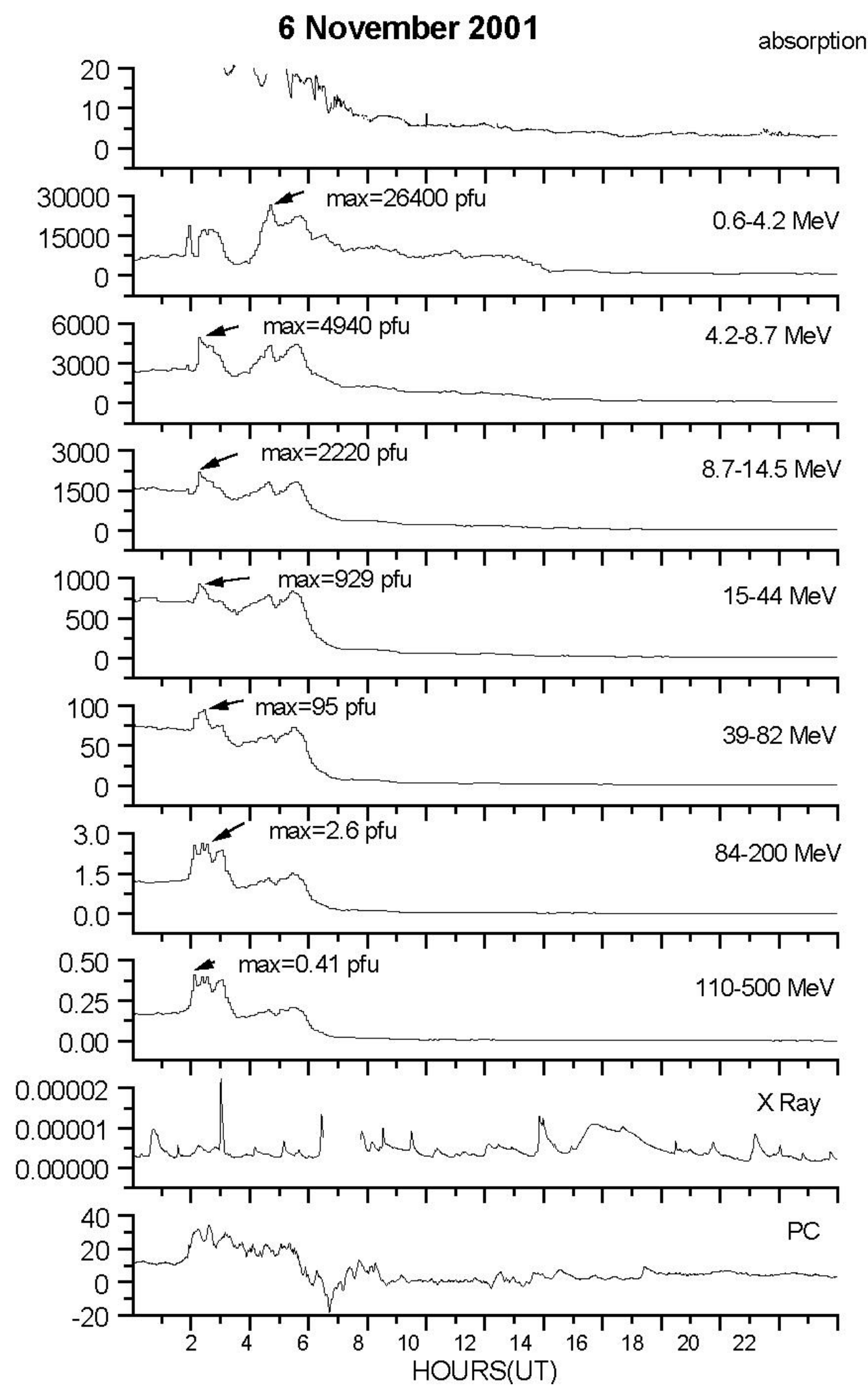

Fig. 4. As Fig. 1, but for 6 November. 


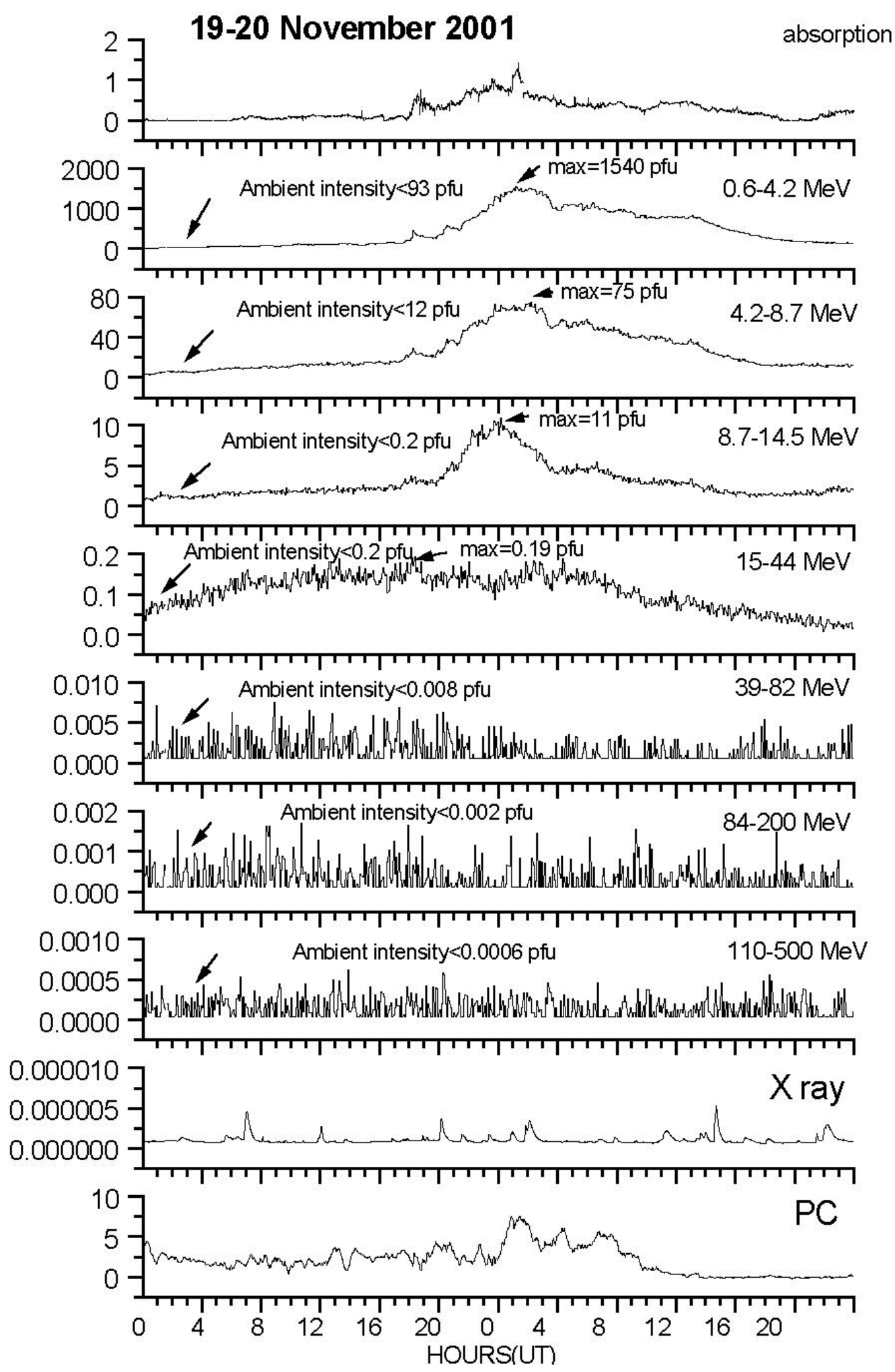

Fig. 5. As Fig. 1, but for 19-20 November. 


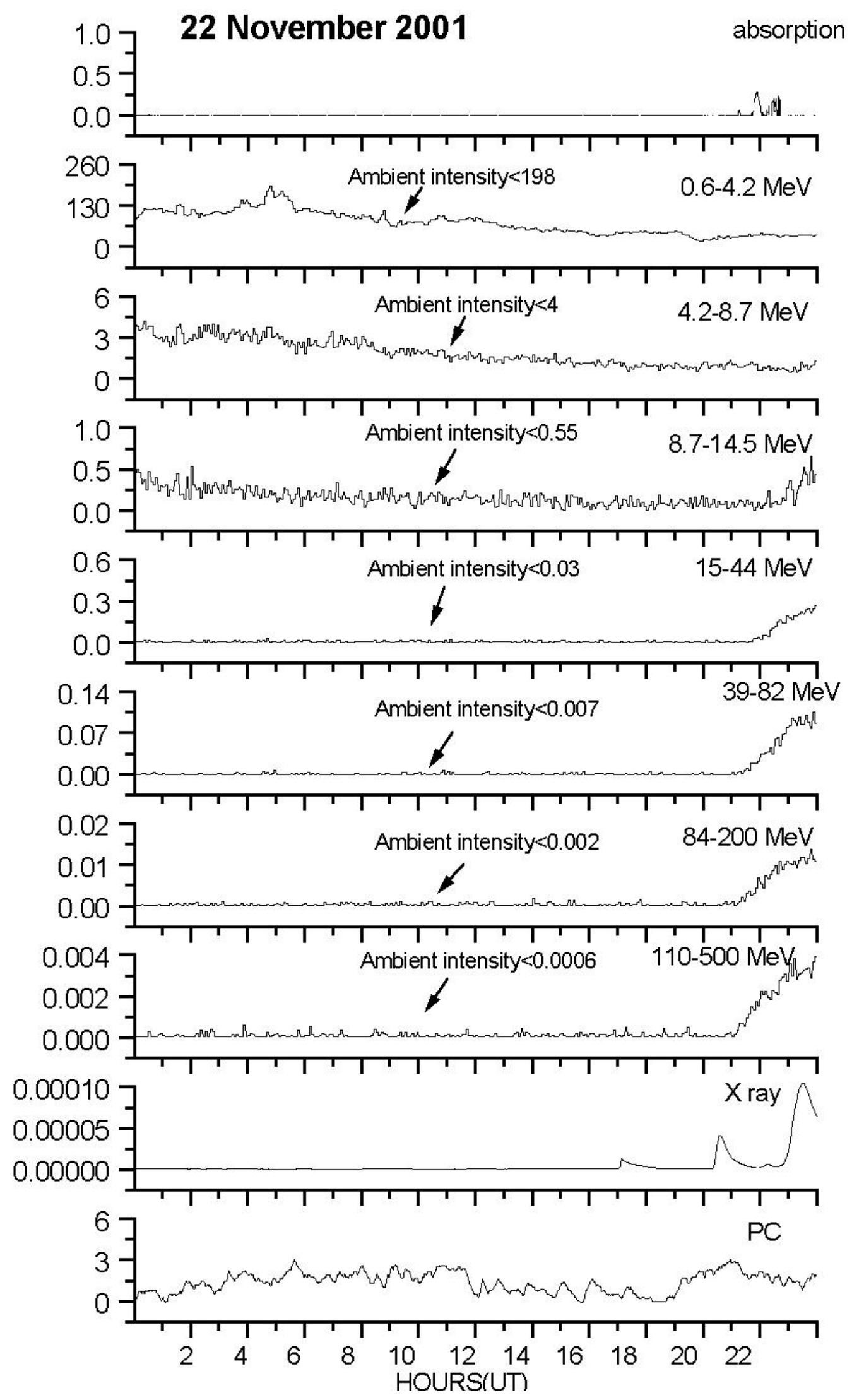

Fig. 6. As Fig. 1, but for 22 November. 


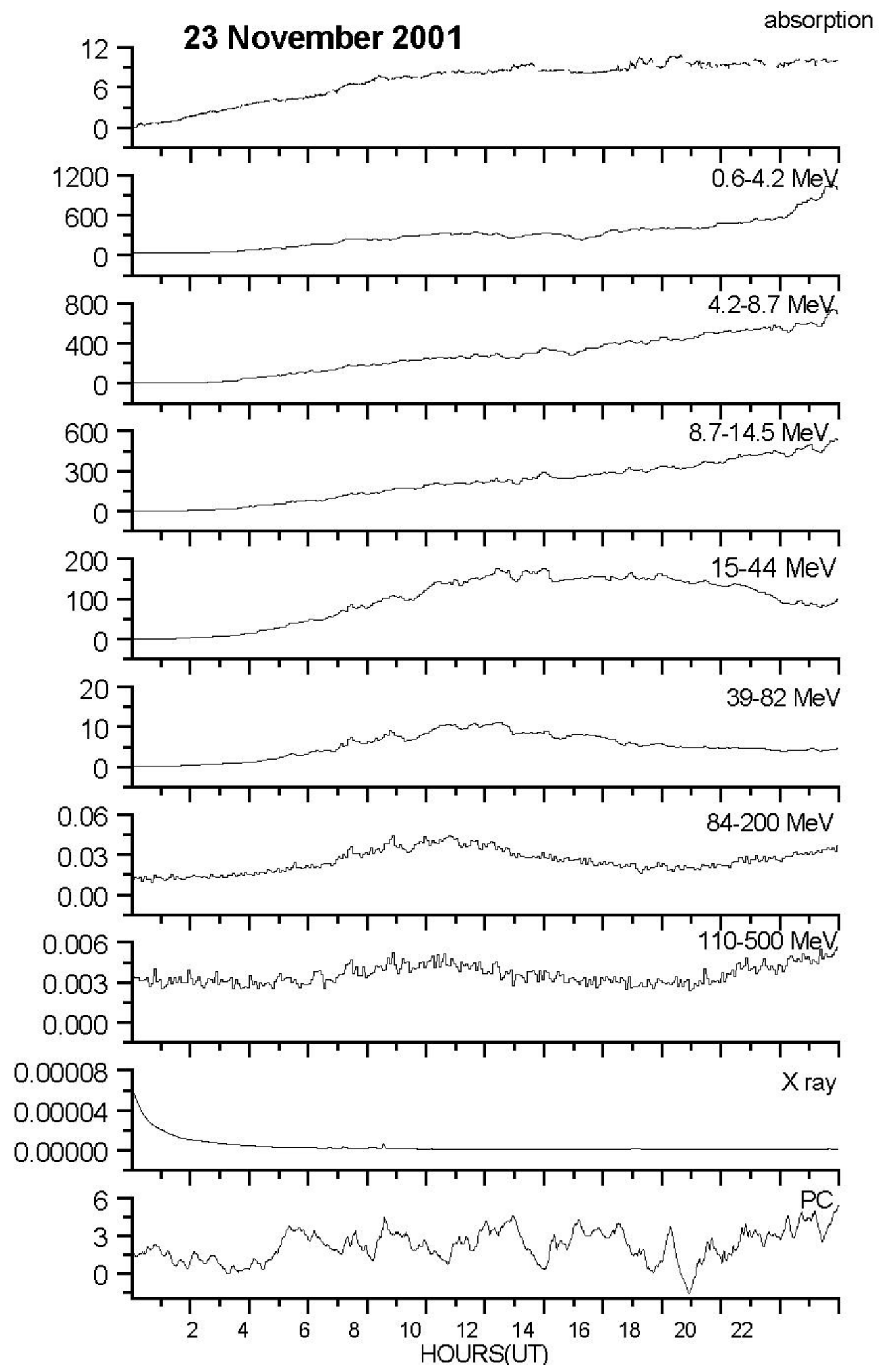

Fig. 7. As Fig. 1, but for 23 November. 


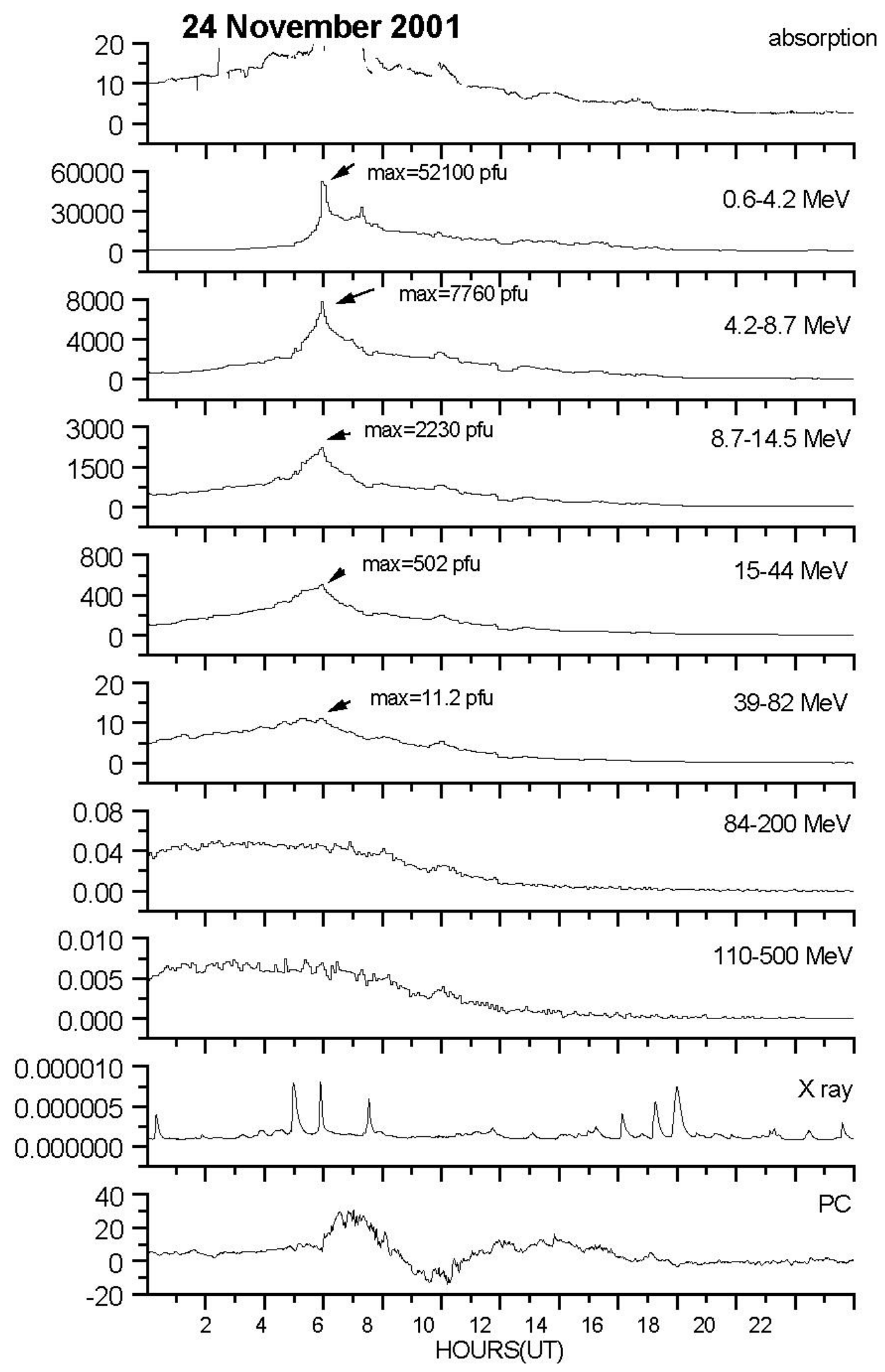

Fig. 8. As Fig. 1, but for 24 November. 


\section{November 2001}

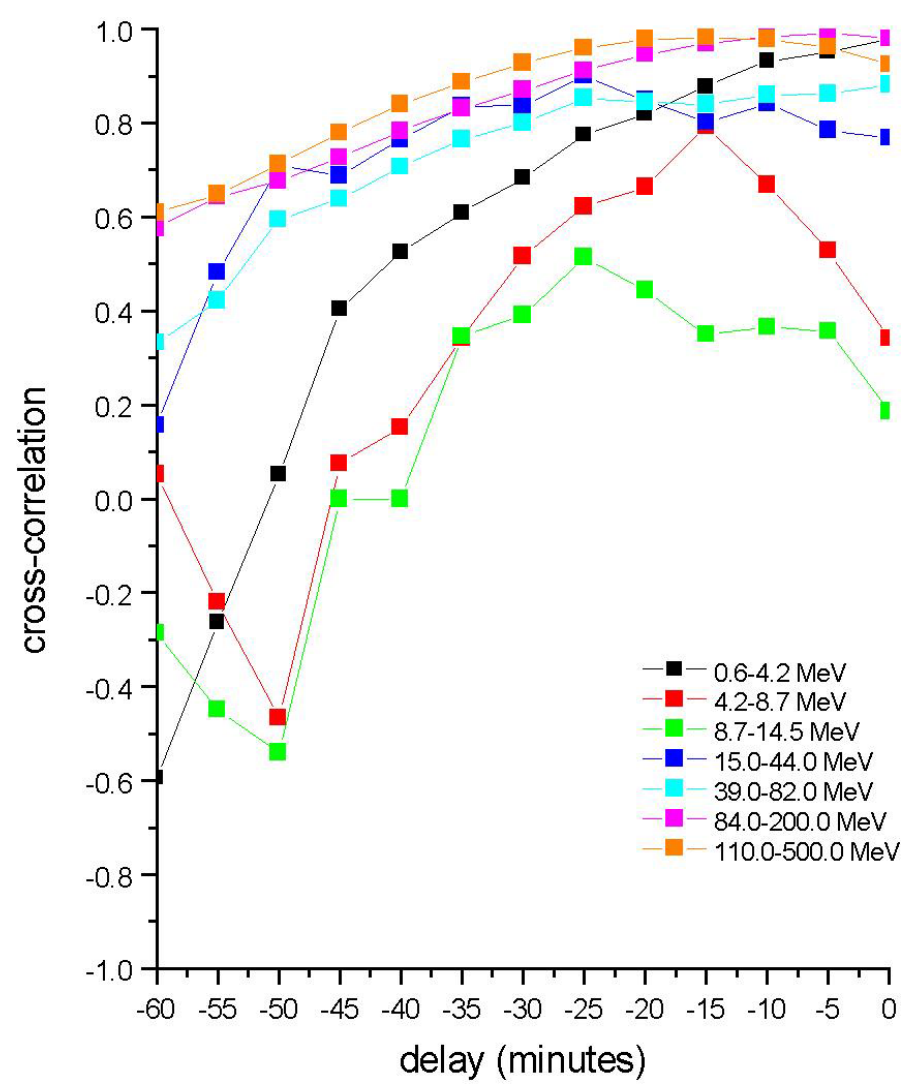

Fig. 9. Cross-correlation coefficients between $A(t)$ and differential $J^{1 / 2}(t-\tau)$ calculated for the first hour of 4 November PCA event (16:55 UT-17:55 UT).

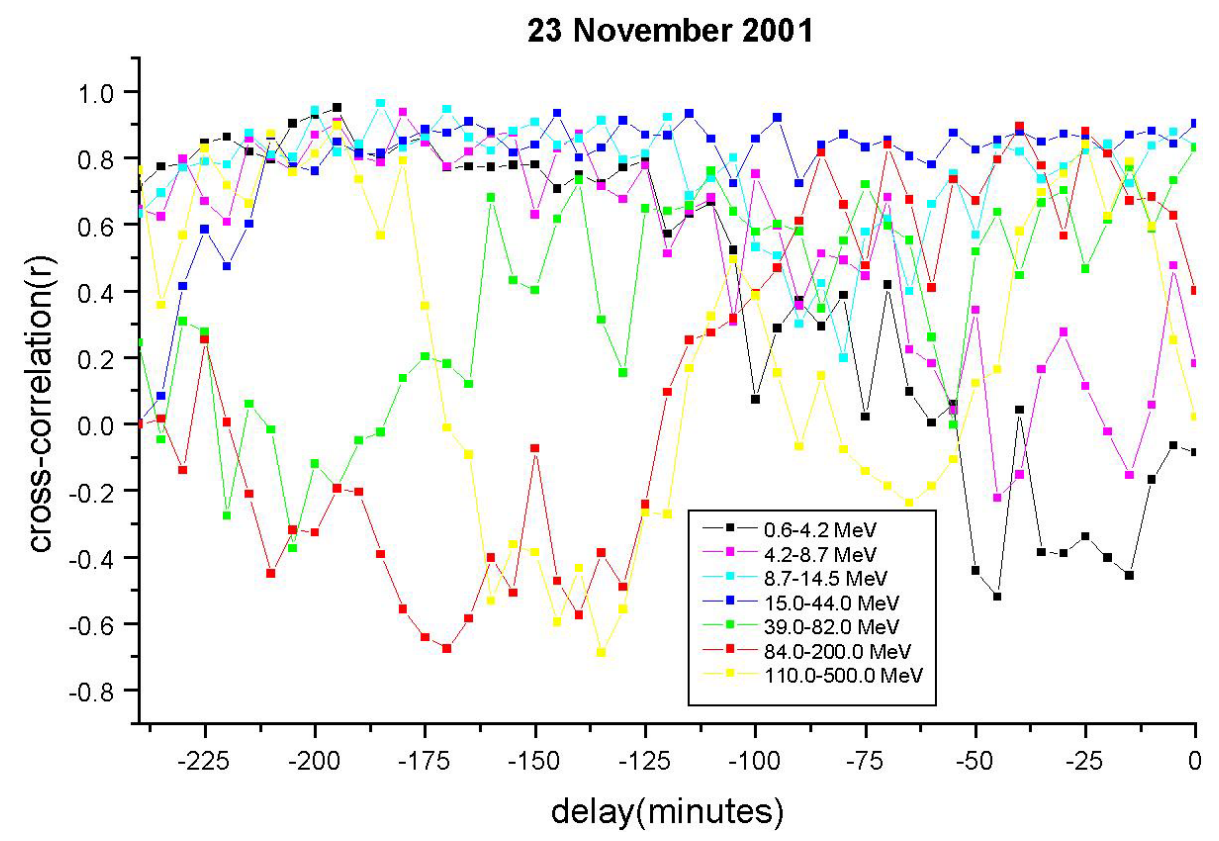

Fig. 10. Cross-correlation coefficients between $A(t)$ and differential $J^{1 / 2}(t-\tau)$ calculated for the first hour of 23 November PCA event (00:25 UT-01:25 UT). 

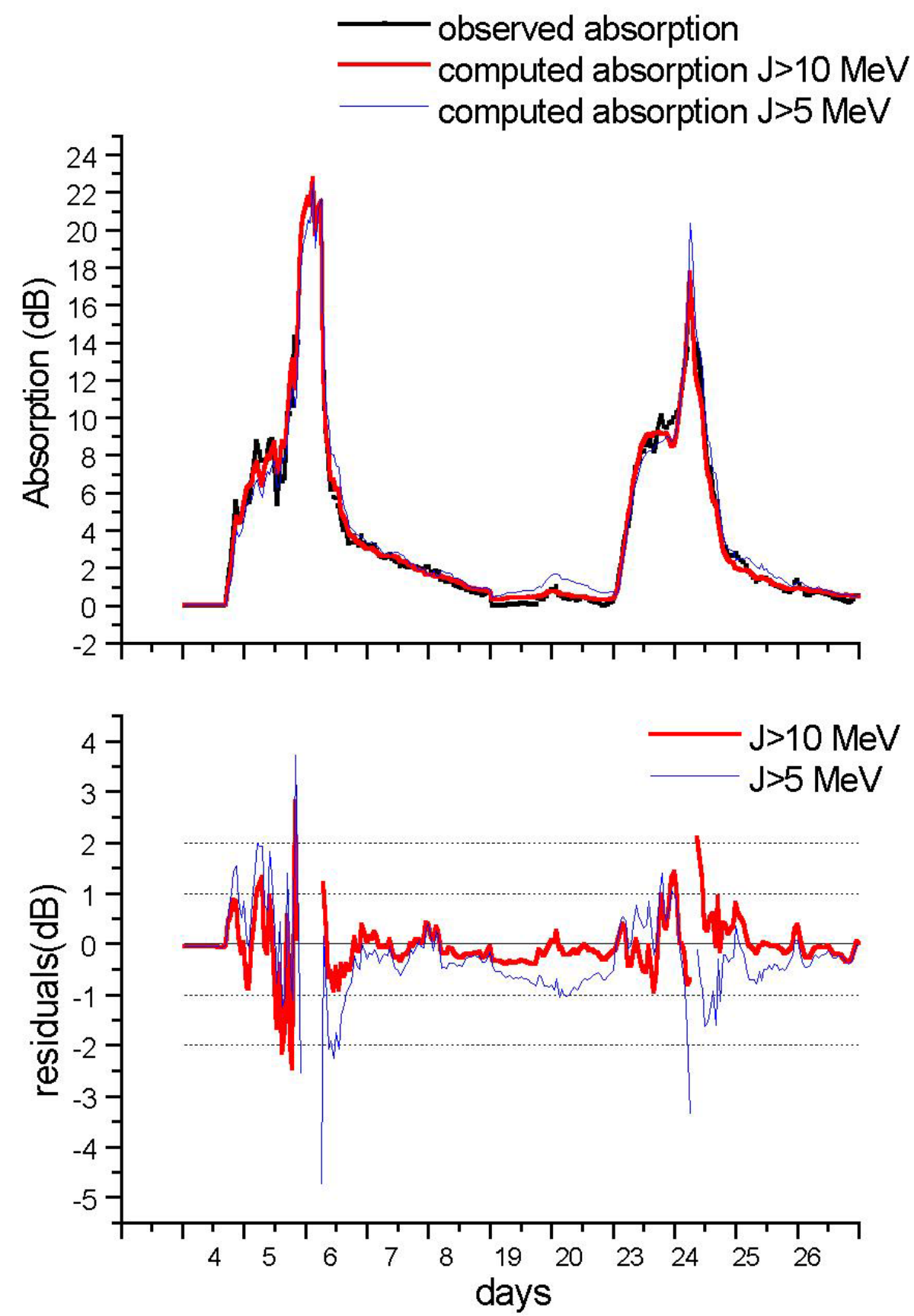

Fig. 11. Hourly means of observed and computed absorption (for $J>5 \mathrm{MeV}, J>10 \mathrm{MeV}$ ) for the three PCA events. Corresponding residuals (observed - calculated absorption) are also plotted.

protons of $15-44 \mathrm{MeV}$ contribute mainly to the beginning of PCA, whereas the protons with energy $4.2-14.5 \mathrm{MeV}$ begin to enhance in a successive phase.

\subsection{Testing the relationship between absorption and proton flux during the PCA event}

These good correlations led us to apply a linear fit to the absorption and the square root of integrated solar proton flux, providing a reasonable empirical relationship (e.g.
Potemra, 1972). The slope $m$ and the correlation coefficient $\mathrm{r}$ are determined using the hourly averages of integral solar proton flux. The best correlations are found for $J>5 \mathrm{MeV}(r=0.97)$ with $m=0.11 \mathrm{~dB}\left(\mathrm{~cm}^{2} \mathrm{~s}\right)^{1 / 2}$ and for $J>10 \mathrm{MeV}(r=0.99)$ with $m=0.14 \mathrm{~dB}\left(\mathrm{~cm}^{2} \mathrm{~s}\right)^{1 / 2}$. Figure 11 shows the computed absorption, the observed absorption and the residuals (observed-calculated) for the three PCA events combined into a single time series. Notwithstanding these higher correlations on some occasions, the computed absorption underestimates the observed values, whereas at other 
times it is overestimated. Most of the residuals fall within $\pm 0.5 \mathrm{~dB}$, for $J>5 \mathrm{MeV}$ the residual percentage is $61 \%$ while for $J>10 \mathrm{MeV}$ it is $80 \%$. For the residuals exceeding $\pm 1 \mathrm{~dB}$, when the computed absorption overestimates the observed absorption, the percentage for $J>5 \mathrm{MeV}$ is $34 \%$ while for $J>10 \mathrm{MeV}$ it is $2 \%$. In the case for which the computed absorption underestimates the observed absorption, the percentage for $J>5 \mathrm{MeV}$ is $5 \%$ while for $J>10 \mathrm{MeV}$ it is $4 \%$. So the residuals calculated for $J>10 \mathrm{MeV}$ are smaller than those obtained for $J>5 \mathrm{MeV}$. In particular, the overestimation of the observed absorption is significant in the case of $J>5 \mathrm{MeV}$. The poorest results are obtained for high solar proton fluxes, in particular on 5 November, for which residuals range between 2.5 and $2.8 \mathrm{~dB}$. The low correlation found between the differential solar proton flux and ionospheric absorption (Table 1) is confirmed by these high residuals. For example, the overestimation $(\sim-2.5 \mathrm{~dB})$ obtained with $J>10 \mathrm{MeV}$ is poorer than that obtained for $J>5 \mathrm{MeV}$ $(\sim-1.2 \mathrm{~dB})$, because in this second case it is inserted into the energy band 4.2-8.7 MeV for which there was found the best correlation (Table 1). A reason for the overestimation could be the occurrence of solar radio emission, whereas for the underestimation an X-ray increase is probably responsible; four solar flares of class $\mathrm{M}$ occurred, and the high geomagnetic activity has caused an enhancement of the absorption. The second case for which the errors are significant is between 23 and 24 November for which the residuals $(\sim 2 \mathrm{~dB})$ are positive; the associated geomagnetic activity suggests that softer particles were also involved, enhancing the observed ionospheric absorption.

\section{Discussion and conclusions}

1. The SPEs on 4 November and 22 November are among the dozen most intense events since 1954, with peaks of integrated solar protons flux $(J>10 \mathrm{MeV})$ of 31700 and 18900 counts $/ \mathrm{cm}^{2} \cdot \mathrm{s} \cdot \mathrm{sr}$. They are associated with large solar flares (X1 and M9.9) originating from the Sun's western hemisphere and with high CME speeds. Unlike these great events, the SPE linked to the solar flare of 17 November is less intense $\left(J=34\right.$ counts $\left./ \mathrm{cm}^{2} \cdot \mathrm{s} \cdot \mathrm{sr}\right)$. The peak intensity of an SPE depends on several factors, one of which is the CME speed. The CME of 4 November has a speed of $1810 \mathrm{~km} / \mathrm{s}$, which suggests that it can produce an intense SPE. The CMEs of 22 November - on the other hand - have a speed of about $1440 \mathrm{~km} / \mathrm{s}$, but probably the enhanced ambient SPE population that can be observed in the energy band between $0.6-44 \mathrm{MeV}$ and the interaction of the two CMEs contribute to the enhancement of the peak intensity in the energy bands 0.6-14.5 MeV. The impact of CME with the geomagnetic field for the two events corresponds to the peaks of PCA and of the magnetic activity (PC index). The less intense SPE has a softer spectrum due to the solar source at eastern longitudes, which also results in longer time delays ( $\sim 2$ days) between the beginning of the SPEs and the solar flares, than in the other two events.

2. The 4 November event, when also a Ground Level Enhancement (GLE) was recorded (Richardson et al., 2003), shows a harder spectrum than the 22 November SPE. This is also supported by the protons' energies which contribute predominantly to the absorption. So the starting phase of the absorption for the first event is due mainly to $84-500 \mathrm{MeV}$ and $0.6-4.2 \mathrm{MeV}$, with a time delay of $15, \ldots, 0 \mathrm{~min}$ and a correlation around 0.98 , whereas for the second event the starting phase of the absorption is mainly due to protons with energies between 15 and $44 \mathrm{MeV}$ with $\tau=-210, \ldots, 0 \mathrm{~min}$ and a poorer correlation compared to the earlier event $(r \sim 0.90)$. Considering the entire period, the main contribution of the event of 4 November is due to protons with energies from 4.2 to $82 \mathrm{MeV}$ penetrating to altitudes ranging from 80 to $40 \mathrm{~km}$. For the event of 19 November the main contribution comes from protons with energies 0.6 to $8.7 \mathrm{MeV}$. This means that the primary contribution to the PCA arises from an ionospheric region $>70 \mathrm{~km}$. On the other hand, on 23 November, the main contribution is from protons of 4.2 to $14.5 \mathrm{MeV}$ penetrating to altitudes between 80 and $60 \mathrm{~km}$.

3. The empirical relationship between proton flux and riometer absorption $A \propto J^{1 / 2}$ is applied for $J>5 \mathrm{MeV}$ and $J>10 \mathrm{MeV}$. The best correlations are found for $J>5 \mathrm{MeV}(r=0.97)$ with $m=0.11 \mathrm{~dB}\left(\mathrm{~cm}^{2} \mathrm{~s}\right)^{1 / 2}$ and for $J>10 \mathrm{MeV}(r=0.99)$ with $m=0.14 \mathrm{~dB}\left(\mathrm{~cm}^{2} \mathrm{~s}\right)^{1 / 2}$. The residuals calculated for $>10 \mathrm{MeV}$ are smaller than those obtained for $J>5 \mathrm{MeV}$ and they exceed $\pm 1 \mathrm{~dB}$ in $6 \%$ of the cases. One factor is due to the chosen threshold ( $E=10 \mathrm{MeV}$ ) for which significant contributions by the lower energy protons are neglected. The others factors which may affect the result could be due to the overestimation by the occurrence of solar radio emission, whereas for the underestimation the X-ray increase and the high geomagnetic activity have probably caused an enhancement of the absorption.

Acknowledgements. The authors would like to thank the Italian National Program of Antarctic Researches (PNRA) for supporting this study, the Space Environment Center (NOAA, US Dept. of Commerce, Boulder, CO, USA) and the Danish Meteorological Institute for providing data, the referees for improving the manuscript.

Topical Editor M. Lester thanks R. Morris and M. Friedrich for their help in evaluating this paper.

\section{References}

Cane, H. V., Erickson, W. C., and Prestage, N. P.: Solar flares, type III radio bursts, coronal mass ejections, and energetic particles, J. Geophys. Res., 107, 14-1, 14-19, 2002.

Cane, H. V., von Rosenvinge, T. T., Cohen, C. M. S., and Mewaldt, R. A.: Two components in major solar particle events, Geophys. Res. Lett., 30, 8017-8020, 2003. 
Chivers, H. J.: High latitude ionospheric absorption, Antarc. J.U.S., X, 222-223, 1975.

Collis, P. N. and Rietveld, M. N.: Mesospheric observations with the EISCAT UHF radar during polar cap absorption events: electron densities and negative ions, Ann. Geophys., 8, 809-824, 1990.

Davies, K.: Ionospheric Radio, Peter Peregrinus Ltd., London, UK, 1990.

De Franceschi, G., de Santis, A., and Perrone, L.: Analysis of Riometric data recorded at Terra Nova Bay, Antarctica, Conference Proceedings of Solar Terrestrial Predictions Workshop, edited by Heckman, G., Marubashi, K., Shea, M. A., Smart, D. F., and Thompson, R., RWC Tokyo, 431-434, 1997.

Hargreaves, J. K., Shirochkov, A .V., and Farmer, A. D.: The polar cap absorption event of 19-21 March 1990: recombination coefficients, the twilight transition and midday recovery, J. Atmos. Terr. Phys., 55, 857-862, 1993.

Kahler, S. W.: The correlation between solar energetic particle peak intensities and speeds of coronal mass ejections: Effect of ambient particle intensities and energy spectra, J. Geophys. Res., 106(A10), 20 947-20 955, 2001.

Little, C. G. and Leinbach, H.: The riometer: a device for the continuous observation of ionospheric absorption, Proc. I.R.E., 47, 315-320, 1959.

Lukianova, R. and Troshichev, O.: The polar cap magnetic activity indices in the southern (PCS) and northern (PCN) polar caps: Consistency and discrepancy, Geophys. Res. Lett, 29, 18791782, 2002.

Patterson, J. D., Armstrong, T. P., Lird, C. M., Detrick, D. L., and Weatherwax, A. T.: Correlation of solar energetic protons and polar cap absorption, J. Geophys. Res., 106, 149-163, 2001.

Pietrella, M., Perrone, L., Romano, V., Alfonsi, L., Cerrone, M., and Rossi, C.: Upper atmosphere effects of solar and magnetic perturbations over Antarctica, ESA, SP-477, 471-474, 2002.
Potemra, T. A.: The empirical connection of riometer absorption to solar protons during PCA events, Radio Sci., 7, 571-577, 1972.

Ranta, H., Ranta, A., Yousef, S. M., Burns, J., and Stauning, P.: D-region observations of polar cap absorption events during the EISCAT operation in 1981-1989, J. Atmos. Terr. Phys, 55, 751766, 1993.

Ranta, H., Yamagishi, H., and Stauning, P.: Twilight anomaly, midday recovery and cutoff latitudes during the intense polar cap absorption event of March 1991, Ann. Geophys., 1, 262-276, 1995.

Reames, D. V.: Particle acceleration at the Sun and in the heliosphere, Space Sci. Rev., 90, 413-491, 1999.

Reid, G. C. and Collis, C.: Observations of abnormal VHF radio wave absorption at medium and high latitudes, J. Atmos. Terr. Phys., 14, 68-81, 1959.

Reid, G. H.: Polar Cap Absorption - Observations and Theory, Fundamentals of Cosmic Physics, 1, 167-204, 1974.

Richardson, I. G., Lawrence, G. R., Haggerty, D. K., Kucera, T. A., and Szabo, A.: Are CME "interactions" really important for accelerating major solar energetic particle events?, Geophys. Res. Lett., 30, 14, 1763-1766, 2003.

Sellers, B., Hanser, F. A., Stroscio, M. A., and Yates, G. K.: The night and day relationships between polar cap riometer absorption and solar protons, Radio Sci., 5, 779-789, 1977.

Stauning, P.: High-Latitude D-and E-region investigations using imaging riometers observations, J. Atmos. Sol. Terr. Phys., 586, 765-783, 1996.

Van Allen, J. A., Lin, A. W., and Leinbach, H.: On the relationship between absolute solar cosmic ray intensity and riometer absorption, J. Geophys. Res., 69, 4481-4491, 1964.

Van Allen, J. A., Fennell, J. A., and Ness, N. F.: Asymmetric access of energetic solar protons to the Earth's north and south polar caps, J. Geophys. Res., 76, 4262-4275, 1971. 